\title{
Editorial
}

Visceral Medicine

\section{Alcohol Use and Gastrointestinal Diseases}

\author{
Helmut Karl Seitz ${ }^{a}$ Hans Scherübl ${ }^{b}$ \\ a Medizinische Klinik (Schwerpunkt Gastroenterologie) Krankenhaus Salem und St. Vincentius Hospital (Schwerpunkt

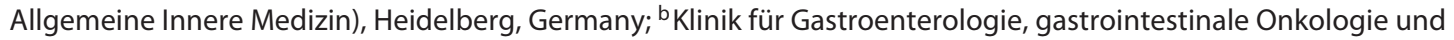 \\ Infektiologie, Vivantes Klinikum Am Urban, Akademisches Lehrkrankenhaus der Charité Berlin, Berlin, Germany
}

According to the World Health Organization (WHO), alcohol use is responsible for more than 200 diseases including accidents and suicides. In 2012, 2.3\% of all deaths in Germany were attributed to alcohol intake [1]. According to recent data from the German Cancer Research Centre (DKFZ), more than $50 \%$ of all alcohol-caused deaths for both males and females are due to gastrointestinal diseases. The most frequent alcohol-attributable fractions for selected causes of death are liver cirrhosis with $50 \%$, pancreatitis with $25 \%$, and esophageal cancer with $22 \%$ [2]. This means that about $50 \%$ of the cirrhosis deaths and 500,000 annual deaths in Europe are due to alcohol use. Thus, there are a lot of reasons why to discuss this topic with international experts in this special issue of Visceral Medicine.

As pointed out above, alcoholic liver disease (ALD), pancreatitis, and cancers are the most important diseases caused by alcohol use. Therefore, epidemiology, clinical features, diagnostics, and therapy for these alcohol-mediated diseases are focused on in this review [2]. Over the last decades, it has been shown that the susceptibility towards alcohol and the risk of disease depend not only on the amount of alcohol consumed but also on the target organ as well as on genetic and non-genetic risk factors. The more alcohol is consumed, the higher the risk of organ damage. The most sensitive organ with respect to alcohol exposure is the female breast, and for alcohol-mediated breast cancer no threshold dose exists. Although not addressed in any detail in this issue, it should be mentioned that early alcohol drinking in life at the age of 12-
15 years increases breast cancer risk in later life significantly [3].

Indeed, one of the most underestimated diseases associated with chronic alcohol misuse is cancer. Worldwide, more than $6 \%$ of all cancers are due to alcohol drinking. This includes cancers of the oral cavity, pharynx, larynx, female breast, esophagus, stomach, liver, colorectum, and possibly pancreas. The article by Scherübl [4] has focused on this important topic discussing mechanisms and prevention strategies. In this context, it is noteworthy to say that genetic risk factors do modify cancer risk, in particular genes coding for enzymes involved in ethanol metabolism such as alcohol dehydrogenase $(\mathrm{ADH})$ and acetaldehyde dehydrogenase (ALDH). Ethanol metabolism occurs not only in the liver but also in the gastrointestinal mucosa. Acetaldehyde is the first, most toxic and carcinogenic metabolite of ethanol. Whenever it accumulates, the risk for upper gastrointestinal cancer increases. Some persons exhibit an enhanced oxidation of ethanol due to a polymorphism of $A D H 1 C$, and others up to $40 \%$ of the population in several Asian countries have a poor metabolism of acetaldehyde due to a mutation of $A L D H 2$. In both situations, acetaldehyde accumulates in the saliva, and cancer risk rises. Acetaldehyde is also present in tobacco smoke, but most often acetaldehyde is produced from oral bacteria. The paper by Salaspuro [5] was one of the first to describe bacterial ethanol metabolism.

Common gastrointestinal cancers associated with heavy alcohol intake include cancers of the large intestine and the 
pancreas. Grumish et al. [6] from Rush University, Chicago, IL, have focused on this topic with new results emphasizing the diurnal rhythm involved in immunology of the colon and its sensitivity towards alcohol. Risk factors for alcoholmediated colorectal carcinogenesis include again acetaldehyde produced by bacteria and oxidative stress possibly generated by ethanol-induced mucosal cytochrome P4502E1 under certain conditions. New data emphasizes the role of the microbiota not only for the colon but also for ALD, since endotoxins may enter the portal system through a „leaky“ gut and may finally end up in the liver.

Alcohol-induced pancreatic cancer risk may be modified by certain mutations predisposing to pancreatitis but also by smoking tobacco or excess body weight. Thus, chronic pancreatitis is associated with an increased risk for pancreatic cancer. Acute and chronic alcoholic pancreatitis is still challenging to treat. Epidemiology, pathophysiology, clinical presentation, and conservative therapy of alcoholic pancreatitis are discussed by Zorniak et al. [7], while surgical therapy of chronic alcoholic pancreatitis is summarized by Miksch et al. [8]. Finally, Desai et al. [9] present new data on fatty pancreas being associated with an increased risk of pancreatic cancer. Glowka et al. [10] provide a very useful update on shunt surgery for portal hypertension.

Without a doubt, the liver is the major target for alcohol. Research results of the last years have identified genetic risk factors. Thus, genetic polymorphisms are important risk factors for liver cirrhosis. Genetic predisposition may explain why only $10-20 \%$ of the heavy drinkers develop cirrhosis. Women have a significantly higher risk for ALD than men. Genetic association studies have identified several genetic risk factors. The most often reported genetic variant is rs738409 in PNPLA3. In addition, an association between rs58542926 (TM6SF2) and rs641738 (MBOAT7) with ALD has been described, while genetic variation in $H S D 17 B 13$ reduces the risk for alcoholic cirrhosis and hepatocellular cancer. All genes so far detected are involved in hepatic fat metabolism [11].

Other risk factors for liver disease include other etiologies such as chronic hepatitis-B or - $\mathrm{C}$ infections, hereditary hemochromatosis, and non-alcoholic steatohepatitis (NASH). Whether moderate alcohol use is beneficial for patients suffering from either non-alcoholic fatty liver disease (NAFLD) or NASH has been a matter of debate. For many years, it has been put forward that moderate alcohol intake might improve peripheral insulin resistance. New data does not argue for alcohol intake in case of NAFLD. However, recent evidence emphasizes not to use alcohol once NASH has been diagnosed. The role of excess body weight, diabetes, and metabolic syndrome in NAFLD is discussed in detail by Malnick and Maor [12].

Other important gastrointestinal aspects of alcohol intake include gastroesophageal reflux disease, severely damaged gastrointestinal mucosa with hemorrhagic gastritis, and lactose intolerance. In addition, alcohol use deteriorates both celiac disease and chronic inflammatory bowel disease and affects gastrointestinal motility. Discussing all alcohol-related gastrointestinal topics may well go beyond the constraints of a focused issue.

Patients with alcohol use disorders who need (emergency) surgery require special attendance. Careful perioperative management of alcohol withdrawal syndrome is necessary to achieve good overall therapeutic success. Therefore, alcohol withdrawal syndrome in this setting has been reviewed by Ungur et al. [13].

The present issue of Visceral Medicine comprising nine articles by experts in the field as well as an interdisciplinary discussion chaired by H.K. Seitz [14] aims to give the reader an updated overview on alcohol use and gastrointestinal disease.

\section{References}

1 World Health Organization. Global status report on alcohol and health 2014. Available from: https://www.who.int/substance_abuse/ publications/global_alcohol_report/en/.

2 Deutsche Krebsforschungszentrum Heidelberg (DKFZ). Alkoholatlas Deutschland 2017. Available from: https://www.dkfz.de/ de/tabakkontrolle/download/Publikationen/sonstVeroeffentlichungen/Alkoholatlas-Deutschland-2017_Doppelseiten. pdf.

3 Seitz HK, Pelucchi C, Bagnardi V, La Vecchia C. Epidemiology and pathophysiology of alcohol and breast cancer: Update 2012. Alcohol Alcohol. 2012 May-Jun;47(3):204-12.

4 Scherübl H. Alcohol Use and Gastrointestinal Cancer Risk. Visc Med. 2020;36(3). DOI: 10.1159/000507232.

5 Salaspuro M. Local Acetaldehyde - Its Key Role in Alcohol-Related Oropharyngeal
Cancer. Visc Med. 2020;36(3). DOI: 10.1159/000507234.

6 Grumish EL, Armstrong AR, Voigt RM, Forsyth CB, Bishehsari F. Alcohol Induced Immune Dysregulation in the Colon Is Diurnally Variable. Visc Med. 2020;36(3):1-8.

7 Zorniak M, Sirtl S, Mayerle J, Beyer G. What Do We Currently Know about the Pathophysiology of Alcoholic Pancreatitis - a Brief Review. Visc Med. 2020;36(3). DOI: 10.1159/000508173.

8 Miksch RC, D'Haese JG, Werner J. Surgical Therapy of Chronic Alcoholic Pancreatitis. Visc Med. 2020;36(3). DOI: 10.1159/000508174.

9 Desai V, Patel K, Sheth R, Barlass U, Sclamberg J, Bishehsari F. Pancreatic Fat Infiltration Is Associated with Higher Risk of Pancreatic Ductal Adenocarcinoma. Visc Med. 2020; 36(3). DOI: 10.1159/000507457.
10 Glowka TR, Kalff JC, Manekeller S. Update on Shunt Surgery. Visc Med. 2020;36(3). DOI: 10.1159/000507125.

11 Lammert F. Genetik und Epigenetik alkoholischer Lebererkrankungen. In: Seitz HK, Mueller S, editors. Alkoholische Leberund Krebserkrankungen. Berlin/Boston: De Gruyter Verlag; 2019. p. 67-80.

12 Malnik S, Maor Y. The Interplay between Alcoholic Liver Disease, Obesity and the Metabolic Syndrome. Visc Med. 2020;36(3). DOI: 10.1159/000507233.

13 Ungur AL, Neumann T, Borchers F, Spies C. Perioperative Management of Alcohol Withdrawal Syndrome. Visc Med. 2020;36(3). DOI: 10.1159/000507595.

14 Seitz HK, Müller S, Tilg H, Canbay A, Homann N, Zizer D. Alcohol Use and Gastrointestinal Disease. Visc Med. 2020;36(3). DOI: $10.1159 / 000507743$. 